\title{
Direct Correlation of MXene Surface Chemistry and Electronic Properties
}

James L. Hart ${ }^{1}$, Kanit Hantanasirisakul ${ }^{1,2}$, Andrew C. Lang ${ }^{1}$, Babak Anasori ${ }^{1,2}$, Yury Gogotsi ${ }^{1,2}$ and Mitra L. Taheri ${ }^{1}$

1. Department of Materials Science \& Engineering, Drexel University, Philadelphia, PA.

2. A.J. Drexel Nanomaterials Institute, Drexel University, Philadelphia, PA.

MXenes are a class of $2 \mathrm{D}$ materials with the chemical formula $\mathrm{M}_{\mathrm{n}+1} \mathrm{X}_{\mathrm{n}} \mathrm{T}_{\mathrm{x}}(\mathrm{M}=$ transition metal element, $\mathrm{X}=\mathrm{C}$ and/or $\mathrm{N}$, and $\mathrm{T}=$ surface termination, e.g. $-\mathrm{O},-\mathrm{OH},-\mathrm{F}$ ) with currently $20+$ members and the potential for many more. Despite their recent discovery in 2011, MXenes have already demonstrated state-of-the-art performance in fields such as electromagnetic interference shielding, chemical sensing, and energy storage[1]. To a large extent, this exceptional performance is due to MXenes' high metallic conductivity. Methods to further improve conductivity, and thus performance, are a central objective of MXene research. A promising approach is through surface chemistry engineering; density functional theory has predicted a strong influence of surface terminations on MXene conductivity[2]. To date, such predications lack experimental validation. Here, we directly correlate MXene surface chemistry and electronic transport through novel microscopy techniques: direct-detection electron energy-loss spectroscopy[3] (EELS) and simultaneous in situ heating (up to $775^{\circ} \mathrm{C}$ ) and electric biasing. Our experiments uncover important chemistry-property relationships which advance our fundamental understanding of MXenes and provide clear guidelines for the optimization of MXene devices.

Three MXenes were studied: $\mathrm{Ti}_{3} \mathrm{C}_{2}, \mathrm{Ti}_{3} \mathrm{CN}$, and $\mathrm{Mo}_{2} \mathrm{TiC}_{2}$. The $\mathrm{MXenes}$ were spray cast[4] onto heating+biasing nanochips[5]. The basic sample morphology is shown in Fig. 1a-c. Heating and biasing experiments were performed with the DENSsolutions Lightning D9+ holder and a JEOL 2100F TEM. EELS experiments were performed with a GIF Quantum and Gatan K2 summit operated in electron counting mode[3].

The results for the $\mathrm{Ti}_{3} \mathrm{CNT}_{\mathrm{x}}$ sample are shown in Figure 1d-g. For this sample, the initial terminations are $-\mathrm{O},-\mathrm{OH}$, and $-\mathrm{F}$, and there is intercalation of $\mathrm{H}_{2} \mathrm{O}$. Theory predicts this MXene to be metallic[6], but to date, this has not been confirmed experimentally. In Fig. 2d, the post-annealing room temperature (RT) resistance is plotted versus the annealing temperature. Figure 2e shows the resistance versus temperature behavior observed during each annealing step. Clearly, annealing resulted in a reduction in sample resistance and a transition from semiconductor-like to metallic transport (as inferred from the sign of $d \mathrm{R} / d \mathrm{~T})$. To understand these changes in electronic transport, we look to in situ EELS data. The O $\mathrm{K}$ edge (Fig. 2f) shows a large decrease in peak ii relative to peak i between RT and $400{ }^{\circ} \mathrm{C}$, indicating loss of $\mathrm{O}$ which is not strongly bonded with $\mathrm{Ti}$, i.e. the loss of intercalated $\mathrm{H}_{2} \mathrm{O}$. Thus the transition from semiconductor-like to metallic transport is attributed to the loss of intercalated $\mathrm{H}_{2} \mathrm{O}$. This finding confirms metallic conductivity in $\mathrm{Ti}_{3} \mathrm{CN}$ and demonstrates that intercalated $\mathrm{H}_{2} \mathrm{O}$ - present in all asprepared MXenes - can induce semiconductor-like behavior in nominally metallic MXenes. At temperatures above $400{ }^{\circ} \mathrm{C}$, the loss of $\mathrm{H}_{2} \mathrm{O}$ is complete, and $-\mathrm{F}$ begins to desorb (Fig. $2 \mathrm{~g}$ ). Thus the decrease in resistance for annealing steps above $400{ }^{\circ} \mathrm{C}$ is attributed to the loss of $-\mathrm{F}$ terminations. This result is the first direct correlation of MXene termination and electronic properties and confirms predications that de-functionalization of $\mathrm{Ti}_{3} \mathrm{CNT}_{\mathrm{x}}$ increases conductivity. In addition to the described $\mathrm{Ti}_{3} \mathrm{CNT}_{\mathrm{x}}$ results, this talk will discuss the behavior of other MXenes and the effect of large organic intercalants. 
We note that the use of direct-detection and electron counting was highly advantageous for this experiment. With the combined energy resolution/field-of-view offered by DD-EELS, we were able to simultaneously observe all relevant elements ( $C$ through $F$ ) while maintaining sufficient energy resolution to detect the onset of structural transitions, e.g. $\mathrm{Ti}_{3} \mathrm{CNT}_{\mathrm{x}} \rightarrow \mathrm{TiO}_{2}$. Additionally, the reduced sensor noise allowed extremely low electron doses to be used, which is important since MXenes are somewhat beam-sensitive[7].

In summary, we have utilized advanced in situ electron spectroscopy techniques to understand chemistry-property relationships in a rapidly emerging family of $2 \mathrm{D}$ materials, MXenes. Our results provide a critical first step in experimentally understanding and controlling MXene surface chemistry for applications ranging from chemical sensors to electromagnetic interference shielding[8].

References:

[1] B. Anasori, M.R. Lukatskaya \& Y. Gogotsi, Nat. Rev. Mat. 2 (2017), p.16098

[2] M. Khazaei et al, J. Mater. Chem. C 5 (2017), p. 2488.

[3] J.L. Hart et al, Sci. Rep. 7 (2017), p. 8243.

[4] K. Hantanasirisakul et al, Adv. Electron. Mater. 2 (2016), p. 1600050.

[5] H.H. Pérez Garza et al, European Microscopy Congress (2016), p. 237.

[6] Y. Xie \& P.R.C. Kent, Phys. Rev. B 87 (2013), p. 235441.

[7] L.H. Karlsson et al, Nanoletters 15 (2015), p. 4955.

[8] JLH, ACL, and MLT acknowledge funding from NSF MRI grant number 1429661. KH, BA, and YG acknowledge funding from the Department of Energy, Office of Basic Energy Sciences. The authors thank DENSsolutions for providing the in situ sample holder for these experiments.
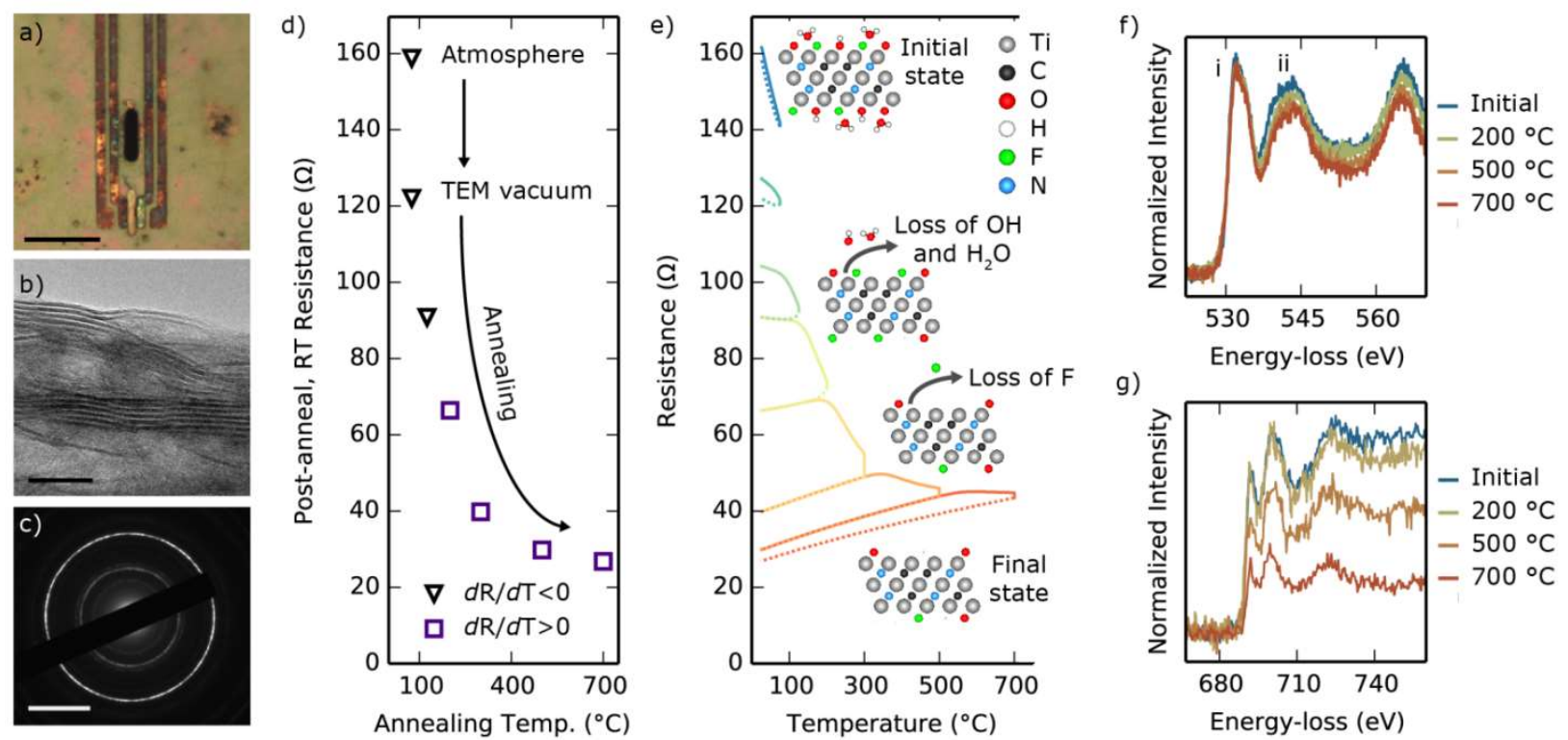

Figure 1. a) Optical image of a MXene film spray cast onto the in situ heating+biasing nanochip, scale bar $=30 \mu \mathrm{m}$. b) TEM image showing the layered structure of $\mathrm{Ti}_{3} \mathrm{CNT}_{\mathrm{x}}$, scale bar $=10 \mathrm{~nm}$. c) Electron diffraction taken after annealing at $700{ }^{\circ} \mathrm{C}$ demonstrating the sample is still MXene, scale bar $=5 \mathrm{~nm}^{-1}$. d,e) Results from in situ heating and biasing within the TEM. Schematics show the changes in MXene intercalation and termination. f,g) In situ DD EELS of the O K and F K edges, respectively. 